\title{
Neumoperitoneo secundario a neumatosis quística intestinal
}

\section{Pneumoperitoneum secondary to intestinal cystic pneumatosis}

\author{
Carlos Tadeo Perzabal Avilez, ${ }^{*}$ César Alberto López Jaime, ${ }^{\ddagger}$ \\ Brian Benito Domínguez González,\$ Diana Laura Carrera Cruz
}

Citar como: Perzabal ACT, López JCA, Domínguez GBB, Carrera CDL. Neumoperitoneo secundario a neumatosis quística intestinal. An Med (Mex). 2020; 65 (4): 311-314. https://dx.doi.org/10.35366/97470

\section{RESUMEN}

La neumatosis quística intestinal es una enfermedad rara, caracterizada por la presencia de quistes aéreos en la pared del tracto gastrointestinal. Su distribución es variable y la localización extraintestinal es poco común. Caso clínico: Mujer de 62 años con dolor abdominal, náusea y vómito. A la exploración abdomen distendido, con dolor a la palpación en hemiabdomen superior, sin datos de irritación peritoneal. Se realizan radiografías de tórax y abdomen, con aire libre subdiafragmático, íleo en mesogastrio y neumobilia, la tomografía con neumatosis quística intestinal de intestino delgado y neumoperitoneo. La neumatosis quística intestinal tiene una prevalencia de $0.03 \%$, tiene una incidencia global de $3 / 10,000$ personas y es más frecuente en hombres que mujeres. Fue descrita por primera vez en 1730 por DuVernoy. Su diagnóstico es complejo, su evolución clínica suele ser crónica y benigna, cursando asintomática en la mayoría de los casos. Los casos con neumatosis quística intestinal representan un reto diagnóstico ante el cirujano, dada la incertidumbre entre el hallazgo imagenológico del aire libre subdiafragmático y la ausencia de datos clínicos de irritación peritoneal.

Palabras clave: Neumatosis quística intestinal, neumoperitoneo, abdomen agudo.

Nivel de evidencia: IV

\begin{abstract}
Cystic intestinal pneumatosis is a rare disease, characterized by the presence of aerial cysts on the wall of the gastrointestinal tract. Its distribution is variable and the extraintestinal location is rare. Clinical Case: A 62-year-old female with abdominal pain, nausea and vomiting. On examination, distended abdomen, with pain on palpation in the upper abdomen, without evidence of peritoneal irritation. X-rays of the chest and abdomen were performed, with subdiaphragmatic open air, ileus in the mesogastrium and pneumobilia, the tomography with cystic small intestine pneumatosis and pneumoperitoneum. Cystic intestinal pneumatosis has a prevalence of $0.03 \%$, an overall incidence of $3 / 10,000$ people and is more frequent in men than women. It was first described in 1730 by DuVernoy. Its diagnosis is complex, its clinical evolution is usually chronic and benign, being asymptomatic in most cases. Patients with cystic intestinal pneumatosis represent a diagnostic challenge before the surgeon, given the uncertainty between the imaging findings of the subdiaphragmatic open air and the absence of clinical data on peritoneal irritation.
\end{abstract}

Keywords: Pneumatosis cystoides intestinalis, pneumoperitoneum, acute abdomen.

Level of evidence: $I V$
* Cirujano General y Laparoscopia, Hospital General de Ciudad Juárez. Chihuahua, México.

‡ Médico Residente de Cirugía General, Hospital General de Ciudad Juárez. Chihuahua, México.

$\S$ Médico Residente de Angiología y Cirugía Vascular, Hospital «Dr. Jesús Enrique Grajeda Herrera». Chihuahua, México.

II Médico General, Hospital General de Ciudad Juárez. Chihuahua, México.
Recibido para publicación: 03/09/2020. Aceptado: 24/11/2020.

Correspondencia: Dr. César Alberto López Jaime Av. Paseo Triunfo de la República Núm. 2401, Margaritas, 32300, Cd. Juárez, Chihuahua. Teléfono: 656-318-52-32

E-mail: cesarlopezj91@gmail.com 


\section{INTRODUCCIÓN}

La neumatosis quística intestinal es una enfermedad rara, caracterizada por la presencia de quistes aéreos en la pared del tracto gastrointestinal. Su distribución es variable, pero es más frecuente en intestino delgado y colon; la localización extraintestinal es poco común. ${ }^{1} \mathrm{Su}$ etiología exacta es desconocida, generalmente va acompañada de enfermedades sistémicas, o relacionada con ingesta de fármacos o traumatismos. Su evolución clínica suele ser crónica y benigna. ${ }^{2}$ El tratamiento depende de la presentación clínica y enfermedades subyacentes, en casos asintomáticos no se precisa tratamiento y en casos leves se recomienda manejo conservador. Normalmente es una condición que no requiere intervención quirúrgica, esta se reserva a casos que presenten síntomas graves o complicaciones. ${ }^{3}$

\section{CASO CLÍNICO}

Se trata de una mujer de 62 años sin antecedentes de importancia, ingresa a urgencias tras presentar cuadro de dolor abdominal en epigastrio, opresivo, moderado que se irradia a hipocondrio izquierdo, acompañándose de náusea y vómito en más de 10 ocasiones.

A la exploración física cardiopulmonar sin compromiso, abdomen globoso a expensas de panículo adiposo, ligeramente distendido, con dolor a la palpación superficial y profunda en hemiabdomen superior, sin datos de irritación peritoneal y con peristalsis hipoactiva.

Se realizan estudios de laboratorio con elevación de neutrófilos, sin leucocitosis, con trombocitosis y elevación de segmentados, así como un descontrol

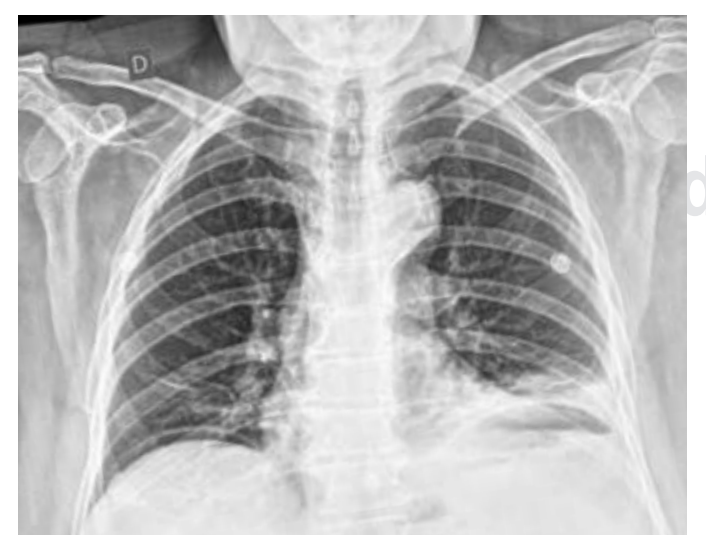

Figura 1: Radiografía de tórax con aire libre subdiafragmático.

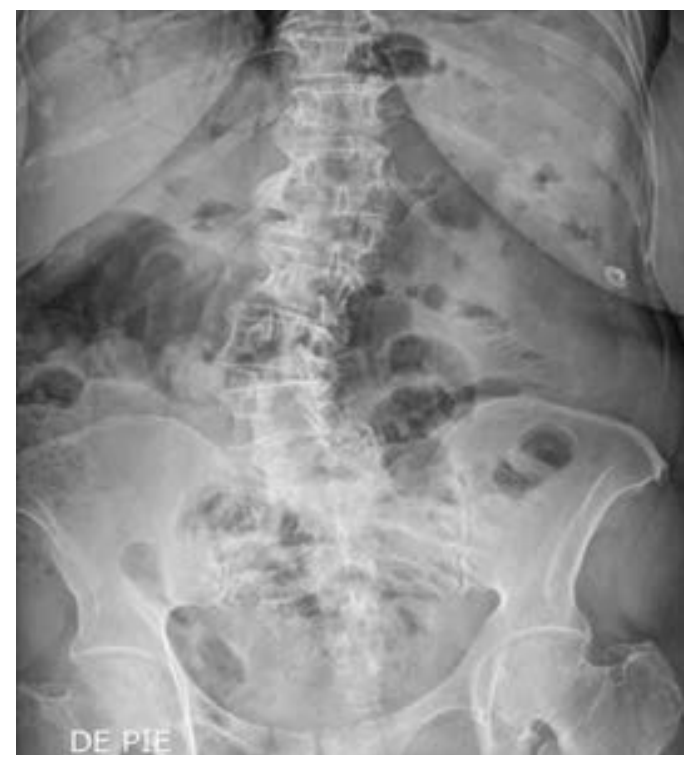

Figura 2: Radiografía de abdomen con neumobilia e íleo reflejo en mesogastrio.

glucémico, sin evidencia de alteración en otros estudios bioquímicos.

Se complementa protocolo diagnóstico con base en radiografías de tórax y abdomen, con presencia de aire libre subdiafragmático (Figura 1), íleo reflejo en mesogastrio y neumobilia (Figura 2), por lo cual se solicita tomografía con hallazgos en relación a neumatosis quística intestinal de intestino delgado, neumobilia, neumoperitoneo y distensión de asas intestinales a nivel de delgado (Figura 3).

Se inicia tratamiento médico conservador con ayuno terapéutico, colocación de sonda nasogástrica, analgésicos, antibioticoterapia y control glucémico, obteniendo respuesta favorable tanto clínica como laboratorial, se inicia de manera paulatina la vía oral y se realiza nueva tomografía abdominal al cuarto día, con mejoría comparativa al estudio previo (Figura 4).

Se egresa a la paciente al quinto día para continuar de manera externa seguimiento por el Servicio de Cirugía General y Medicina Interna.

\section{DISCUSIÓN}

La neumatosis quística intestinal es una enfermedad muy rara, se define como un hallazgo radiológico de gas dentro de la pared del tracto gastrointestinal, caracterizada por quistes aéreos subserosos o submucosos con tamaño variable. ${ }^{1,2}$ La neumatosis quística intestinal afecta típicamente al intestino grueso en $46 \%$ de los casos, e intestino delgado en un $27 \%$, y la 
afección de ambos se da en un $7 \% .{ }^{4}$ Su prevalencia es de $0.03 \%$, tiene una incidencia global de $3 / 10,000$ personas y es más frecuente en hombres que mujeres (proporción 3:1), con un pico de incidencia entre los 25 y 60 años de edad. ${ }^{5}$ Fue descrita por primera vez en 1730 por DuVernoy, y hasta 1835 Mayer utilizó el término neumatosis cistoides intestinorum para describirla. ${ }^{6}$ De manera clásica, se divide en dos tipos: causa primaria o idiopática ( $15 \%$ de los casos) y secundaria $(85 \%)$ en relación con enfermedades sistémicas. ${ }^{7} \mathrm{Su}$ etiología es desconocida, y se cree que es de origen multifactorial, asociada con numerosas patologías como isquemia intestinal, enfermedad pulmonar obstructiva crónica, obstrucción intestinal, enfermedades autoinmunes, inmunosupresión y enfermedad de Crohn. ${ }^{6}$

Se han propuesto diversas teorías para su fisiopatología: teoría mecánica, pulmonar y bacteriana, siendo la última la más aceptada. La teoría mecánica se basa en el paso de gas intraluminal, precipitado por daño en la mucosa por inflamación o necrosis, al haber aumento de la presión intraluminal..$^{1,6,7} \mathrm{La}$ teoría pulmonar sugiere que una ruptura alveolar resulta en disección de aire a través del retroperitoneo hacia mediastino, llegando a la serosa intestinal a través del mesenterio. La teoría bacteriana propone la producción de gas intramural (especialmente hidrógeno) y su difusión a través de la mucosa gástrica se da por una alteración que favorece el crecimiento de bacterias anaerobias. ${ }^{6}$

$\mathrm{Su}$ diagnóstico es complejo, se realiza mediante endoscopia, radiografía simple de abdomen o tomografía computada. Deben excluirse múltiples causas, en particular isquemia intestinal o perforación de alguna víscera hueca. ${ }^{1}$ En la tomografía computarizada, hasta en un tercio de los casos hay neumoperitoneo asociado, el cual puede ser asintomático o llevar a sospechar riesgo de complicación. ${ }^{1}$
Su evolución clínica suele ser crónica y benigna, cursando asintomática en la mayoría de los casos, mientras que los casos con enfermedad que amenaza la vida presentan síntomas agudos. ${ }^{8}$

En la actualidad, no hay tratamiento médico específico, ${ }^{7,9}$ entre las opciones de manejo conservador para casos leves se encuentran: descompresión nasogástrica, oxígeno hiperbárico, antibioticoterapia y escleroterapia. ${ }^{8-10}$ Se debe tener en cuenta que la presencia de neumoperitoneo no implica necesidad de cirugía inmediata. ${ }^{10} \mathrm{El}$ tratamiento quirúrgico se reserva a casos de presentación aguda o complicación como perforación, obstrucción, vólvulos, peritonitis o sepsis abdominal. ${ }^{6}$ Se debe optar por un manejo individualizado, desde laparoscopia en casos estables, hasta laparotomía con colectomía radical. En las formas secundarias, el tratamiento es específico de la enfermedad de base causante. ${ }^{6,8,9}$

\section{CONCLUSIÓN}

Los casos con neumatosis quística intestinal representan un reto diagnóstico ante el cirujano, dada la incertidumbre entre el hallazgo imagenológico del aire libre subdiafragmático y la ausencia de datos clínicos de irritación peritoneal que conllevan; sin embargo, ante un individuo sin evidencia clínica de datos de irritación peritoneal y con hallazgos imagenológicos de neumoperitoneo, siempre se debe indagar más en el diagnóstico, requiriendo la realización de estudios más especializados tales como endoscópicos y/o tomografías computarizadas. De manera acertada en este caso, se optó por la realización de un estudio tomográfico de seguimiento al cuarto día de evolución, el cual reveló mejoría en cuanto a la cantidad del neumoperitoneo residual, sin mencionar la evidente mejoría clínica de la paciente. Ante la duda diagnóstica y el bajo nivel de incidencia de
Figura 3:

\footnotetext{
Tomografía de abdomen en corte axial al ingreso con neumoperitoneo, neumobilia y neumatosis quística intestinal.
}
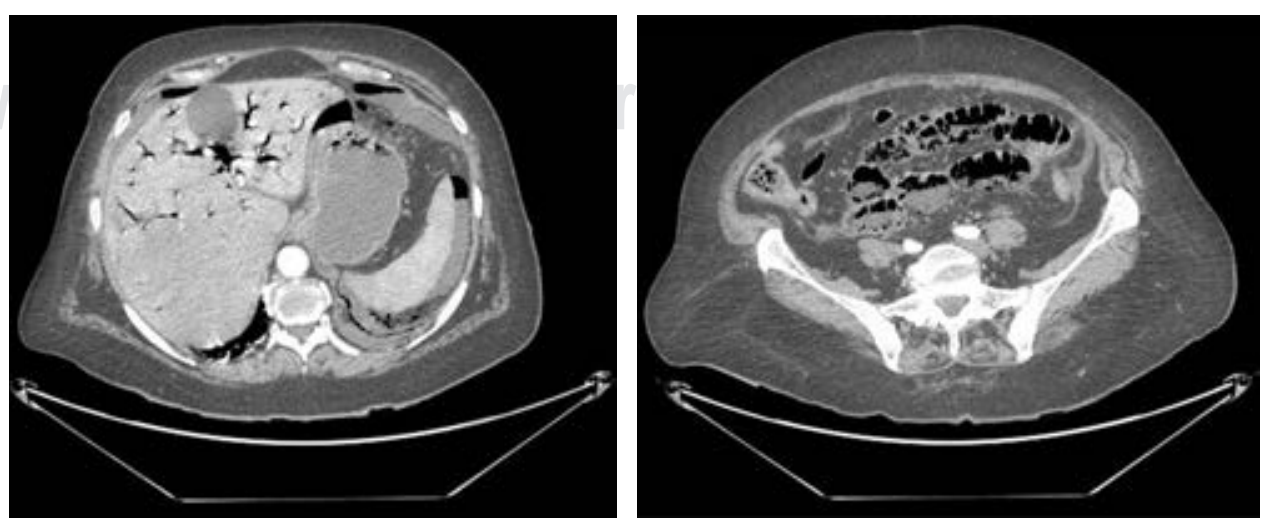

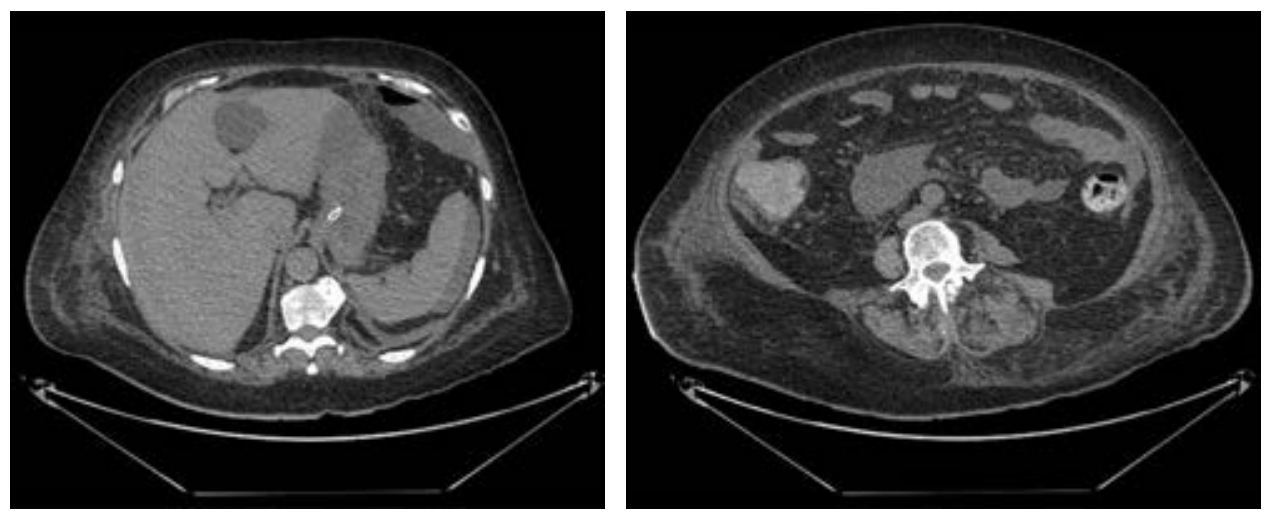

Figura 4:

Tomografía de abdomen en corte axial al cuarto día de estancia hospitalaria con resolución de la neumobilia y de la neumatosis intestinal, persistiendo con escaso neumoperitoneo.

la neumatosis quística intestinal, se puede realizar una laparoscopia diagnóstica o una laparotomía exploradora para descartar las múltiples patologías que ocasionan abdomen agudo y neumoperitoneo.

\section{AGRADECIMIENTOS}

Queremos agradecer y reconocer al personal del Hospital General de Ciudad Juárez por la colaboración y su apoyo en la preparación de este caso.

\section{BIBLIOGRAFÍA}

1. Sellés-Dechent R, Zumárraga-Navas P, Ruiz-Del Castillo J. Neumoperitoneo debido a neumatosis quística intestinal. Ci Esp. 2004; 76 (6): 396-399.

2. Cuevas-Castillejos JE, Valdovinos-Díaz MA. Neumatosis quística intestinal: una causa rara y benigna de dolor y distensión abdominal crónicos con neumoperitoneo. Rev Gastroenterol Mex. 2019; 84 (3): 402-404.

3. Klimova K, Pérez Valderas MD, Merino Rodríguez B, González Asanza C, Menchén Fernández-Pacheco P. Neumatosis quística intestinal como causa infrecuente de dolor abdominal crónico. Rev Gastroenterol Mex. 2014; 79 (4): 302-303.

4. Jenkins M, Courtney H, Pope E, Williamson J. A case report and approach to management in pneumatosis intestinalis. Ann Med Surg. 2017; 23: 25-27.

5. González-Rodríguez F, Puñal-Rodríguez J, Paredes-Cotoré J, Beiras A. Neumoperitoneo espontáneo secundario a neumatosis quística intestinal. Cir Esp. 2009; 86 (2): 111-121.

6. Dhadlie S, Mehanna D, McCourtney J. Pneumatosis intestinalis a trap for the unwary: case series and literature review. Int J Surg Case Rep. 2018; 53: 214-217.

7. Itazaki Y, Tsujimoto H, Ito N, Horiguchi H, Nomura S, Kanematsu K et al. Pneumatosis intestinalis with obstructing intussusception: a case report and literature review. World J Gastrointest Surg. 2016; 8 (2): 173-178.

8. Zuluaga-Santamaría A, MejíaRestrepo J, Uribe-González R, Gutiérrez-Márquez C, Bustamante-Zuluaga C, VegaArango M. Neumatosis intestinal: ¿una urgencia abdominal? Rev CES Med. 2017; 31 (1): 110-118.

9. Donovan S, Cernigliaro J, Dawson N. Pneumatosis intestinalis: a case report and approach to management. Case Rep Med. 2011; 2011: 571387.

10. Asquel Cadena VH, Tovar Noroña CE. Neumatosis quística intestinal, como causa infrecuente de neumoperitoneo. Mediciencias UTA. 2020; 4 (1): 21-27. 